\title{
Generation of Human Melanocytes from Induced Pluripotent Stem Cells
}

\author{
Shigeki Ohta ${ }^{19}$, Yoichi Imaizumi ${ }^{29}$, Yohei Okada, ${ }^{2,3}$, Wado Akamatsu², Reiko Kuwahara', Manabu \\ Ohyama $^{4}$, Masayuki Amagai ${ }^{4}$, Yumi Matsuzaki ${ }^{2}$, Shinya Yamanaka ${ }^{5,6}$, Hideyuki Okano ${ }^{2 *}$, Yutaka \\ Kawakami ${ }^{1 *}$
}

1 Division of Cellular Signaling, Institute for Advanced Medical Research, Keio University School of Medicine, Tokyo, Japan, 2 Department of Physiology, Keio University School of Medicine, Tokyo, Japan, 3 Kanrinmaru Project, Keio University School of Medicine, Tokyo, Japan, 4 Department of Dermatology, Keio University School of Medicine, Tokyo, Japan, $\mathbf{5}$ Center for iPS Cell Research and Application (CiRA), Kyoto University, Kyoto, Japan, 6 Institute for Integrated Cell-Material Sciences, Kyoto University, Kyoto, Japan

\begin{abstract}
Epidermal melanocytes play an important role in protecting the skin from UV rays, and their functional impairment results in pigment disorders. Additionally, melanomas are considered to arise from mutations that accumulate in melanocyte stem cells. The mechanisms underlying melanocyte differentiation and the defining characteristics of melanocyte stem cells in humans are, however, largely unknown. In the present study, we set out to generate melanocytes from human iPS cells in vitro, leading to a preliminary investigation of the mechanisms of human melanocyte differentiation. We generated iPS cell lines from human dermal fibroblasts using the Yamanaka factors (SOX2, OCT3/4, and KLF4, with or without c-MYC). These iPS cell lines were subsequently used to form embryoid bodies (EBs) and then differentiated into melanocytes via culture supplementation with Wnt3a, SCF, and ET-3. Seven weeks after inducing differentiation, pigmented cells expressing melanocyte markers such as MITF, tyrosinase, SILV, and TYRP1, were detected. Melanosomes were identified in these pigmented cells by electron microscopy, and global gene expression profiling of the pigmented cells showed a high similarity to that of human primary foreskin-derived melanocytes, suggesting the successful generation of melanocytes from iPS cells. This in vitro differentiation system should prove useful for understanding human melanocyte biology and revealing the mechanism of various pigment cell disorders, including melanoma.
\end{abstract}

Citation: Ohta S, Imaizumi Y, Okada Y, Akamatsu W, Kuwahara R, et al. (2011) Generation of Human Melanocytes from Induced Pluripotent Stem Cells. PLoS ONE 6(1): e16182. doi:10.1371/journal.pone.0016182

Editor: Huck H. Ng, Genome Institute of Singapore, Singapore

Received August 12, 2010; Accepted December 15, 2010; Published January 13, 2011

Copyright: (c) 2011 Ohta et al. This is an open-access article distributed under the terms of the Creative Commons Attribution License, which permits unrestricted use, distribution, and reproduction in any medium, provided the original author and source are credited.

Funding: The study was supported by a grant from MEXT (Ministry of Education, Culture, Sports, Science and Technology), the project for realization of regenerative medicine and support for the core institutes for iPS cell research from MEXT, and a Grant-in-aid for the Global COE program to Keio University from MEXT. The funders had no role in study design, data collection and analysis, decision to publish, or preparation of the manuscript.

Competing Interests: The authors have declared that no competing interests exist.

*E-mail: hidokano@sc.itc.keio.ac.jp (HO); yutakawa@sc.itc.keio.ac.jp (YK)

9 These authors contributed equally to this work.

- These authors also contributed equally to this work.

\section{Introduction}

Pigmented cells including epidermal melanocytes play an important physiological role in providing protection from harmful ultraviolet rays, and are also implicated in various pigmented cell disorders. Either defects in, or a lack of melanocytes and/or melanocyte stem cells (MELSCs) can lead to pigment disorders such as piebaldism, albinism, vitiligo, and hair graying. Vitiligo is a common disease affecting approximately 0.1 to $2.0 \%$ of the world population, although the pathogenesis has not completely understood [1]. Autologous cultured melanocytes may be useful for the treatment of vitiligo [2,3]. In contrast to foreskin melanocytes, expansion of adult melanocytes is quite difficult. Thus, development of methods to generate large numbers of autologous melanocytes is required. Among pigment cell disorders, melanoma is one of the most aggressive types of human cancers, and is suspected to arise from MELSCs. The recent progress of cancer stem cell studies supports the hypothesis that melanoma stem cells (MMSCs) which are resistant to chemotherapy may exist, and are thus important therapeutic targets [4,5]. It is proposed that MMSCs are generated from MELSCs through accumulation of genetic changes and may have similar phenotypes to MELSCs [6]. Thus, understanding the biology of human MELSCs and MMSCs is critically important. In addition, to permit the investigation of MELSCs and MMSCs, their purification or generation is required. To date, some candidate markers for MELSCs (DCT; dopachrome tautomerase, and PAX3) [7,8] and MMSGs (ABCB5, CD20, CD133, CD271) $[9,10,11,12]$ have been reported, respectively; however, their specificity is still controversial. Refining such knowledge will permit the development of therapeutic treatments against MMSCs, including immunotherapy.

Melanocytes are specialized cells derived from the neural crest cells during embryonic development that migrated to hair follicles and basal layer of the epidermis. A number of studies have shown that cell factors such as MITF, c-Kit, and Snail/Slug are important for melanocyte development. Especially, MITF can regulate the melanocyte lineage in part by regulating several 
pigmentation enzymes including DCT, TYRP1, and tyrosinase [13]. It has been reported that MELSCs locate in the bulge region of hair follicles in mice [7], however, the localization of MELSCis in human skin has been unclear due to lack of definitive markers. It is also difficult to analyze the developmental cell lineages of skin melanocytes in humans. Thus, it is desirable to develop a new in vitro system for generating human melanocytes through MELSCs, which mimics in vivo differentiation processes to better understand human melanocyte development. Melanocyte generation from embryonic stem (ES) cells has been previously reported [14,15]. Alternatively, induced pluripotent stem (iPS) cells have specific advantages compared to ES cells. Besides avoiding ethical issues, iPS cells can be propagated as autologous cells, meaning that melanocytes from autologous iPS cells are not likely to be immunologically rejected if transplanted for the treatment pigment cell disorders. In addition, melanocytes may be generated from the iPS cells of patients with genetic pigment cell disorders, leading the understanding of mechanisms of the diseases, as shown previously for ALS, Familial dysautonomia, Parkinson's disease and SMA $[16,17,18,19]$. With these considerations, human iPS cells are a superior starting cell source to generate melanocytes through neural crest cells in vitro, leading to a better understanding of the characteristics of MELSCs and MMSCs.

In this study, we have established an in vitro system for generating human melanocytes from iPS cells, apparently through a neural crest cell intermediate. This system may contribute to the understanding of human melanocyte development and various pigment cell disorders, including melanoma. It may also be useful for the preparation of large numbers of autologous melanocytes for treating hypopigmental diseases.

\section{Results}

\section{Generation of human iPS cells from human dermal fibroblasts}

We established two human iPS cell lines following the methods established by Takahashi et al. [20] from dermal fibroblasts using all four retrovirally expressed Yamanaka factors (4F) (SOX2, OCT3/4, KLF4, c-MYC) or using three factors (3F) without cMYC (Figure $1 \mathrm{~A}$ and 1B). To examine the expression of pluripotency markers in both $3 \mathrm{~F}$-iPS cells and $4 \mathrm{~F}$-iPS cells, we performed alkaline phosphatase staining (Figure $1 \mathrm{C}$ and 1D) and immunocytochemical analyses on OCT3/4, NANOG, SSEA4, TRA-1-60, and TRA-1-81 in 3F and 4F-iPS cell colonies (Figure 1E- $\mathrm{I}^{\prime}$ ). In addition, the gene expression profiles of pluripotent stem cell markers including the endogenous Yamanaka factors, NANOG and REX1 were analyzed in $3 \mathrm{~F}$ and $4 \mathrm{~F}$-iPS cells by quantitative PCR analyses and compared to 4F-iPS cells' parental human dermal fibroblasts and previously characterized 4F-iPS cells, 201B7 [20]. The generated 3F- and 4F-iPS cells expressed endogenous pluripotency markers similarly to 201B7 iPS cells and ES cells (Figure 1J). Transgene expression was also examined using quantitative PCR analyses, indicating a similarly low gene expression level in the both established 3F- and 4F-iPS cells as compared to 201B7 iPS cells, apart from a higher expression of retroviral KLF4 in 3F-iPS cells (Supplemental Figure $\mathrm{S} 1)$. Evaluation of the methylation status of cytosine guanine dinucleotides $(\mathrm{CpG})$ in the promoter regions of OCT3/4 was analyzed by bisulfite sequencing and compared to $4 \mathrm{~F}$-iPS parental dermal fibroblasts. 3F- and 4F-iPS cells were highly unmethylated in comparison (Figure 1K). Furthermore, the differentiation ability of both 3F- and 4F-iPS cells was examined in vitro. Floating embryoid bodies (EBs) were cultivated for 3 weeks from each iPS cell set, and then spontaneously differentiated onto gelatin coated dishes for 12 days. Attached cells were immunopositive for three germ cell layers' markers ( $\beta$-III-tubulin, ectoderm; $\alpha$-smooth muscle actin, mesoderm; $\alpha$-fetoprotein, endoderm) (Figure 1L$\left.1 \mathrm{~N}^{\prime}\right)$. Finally, to confirm the pluripotency of $4 \mathrm{~F}$-iPS cells in vivo, we transplanted $3 \mathrm{~F}$ - and $4 \mathrm{~F}$-iPS cells into the testis of immunodeficient mice (NOD-SCID) to observe teratoma formation. Tumors formed eight weeks after injection and contained various tissues representing all three germ layers (ectoderm, mesoderm, and endoderm; Figure 1O-1Q') and various tissues (Supplemental Figure S2). Collectively, these results confirmed the establishment of de novo iPS cells from human dermal fibroblasts.

\section{Melanocyte differentiation from human iPS cells}

Human 3F- and 4F-iPS cells were cultured as colonies on mitomycin-C treated feeder cells in iPS medium containing FGF-2 (Figure 2A). To generate melanocytes, EBs were generated from iPS cells grown in suspension in bacterial culture dishes without FGF-2 for 3 weeks (Figure 2B) and then plated onto fibronectincoated dishes in melanocyte differentiation medium containing Wnt3a, SCF, ET3, FGF-2, and cAMP inducers (cholera toxin) as described in Materials and Methods. A week after plating, many cells migrating out from EBs were observed (Figure 2C), and culture was continued for an additional 3 weeks, changing the medium every $2-3$ days. After this period, whole attached cells were dissociated into single cells using TrypLE Select and replated onto a fibronectin-coated dish in the same melanocyte differentiation medium. Proliferating cells were passaged before reaching confluence. Pigmented cells usually appeared 3-4 weeks after single cell dissociation, and most of the cells were highly pigmented $4-6$ weeks after single cell dissociation (Figure 2D, 2E). Using our established system, we can produce approximately $5 \times 10^{6}$ melanocytes cells based on the percentage of SILV ${ }^{+}$cells in the total cell number three months after differentiation from a $10 \mathrm{~cm}$ cell dish containing confluent iPS cell colonies (approximately $2-3 \times 10^{6}$ starting iPS cells). We could usually maintain the culture for a few months. However, proliferative capacity of the cells was eventually lost, as is typically seen in primary human melanocyte culture.

\section{Characterization of human melanocytes derived from iPS cells}

To characterize putative melanocytes derived from both $3 \mathrm{~F}$ and 4F-iPS cells, we performed immunocytochemical analyses using established melanocyte markers. Each set of iPS cell-derived melanocytes was immune-positive for SLIV, TYRP1, TYR, MITF and $\mathrm{S} 100$ antibodies 8 weeks after differentiation (Figure $3 \mathrm{~A}-\mathrm{J}$ ). As a positive control, we analyzed normal human foreskin-derived epidermal melanocyte (NHEM) cells isolated from newborn foreskin using the same antibodies (Figure $3 \mathrm{~K}-\mathrm{O}$ ). To further confirm the generation of melanocytes, melanosome formation in the pigmented cells was evaluated using transmission electron microscopy, revealing melanosomes in both $3 \mathrm{~F}$ - and $4 \mathrm{~F}-\mathrm{PPS}-$ derived melanocytes (Figure $3 \mathrm{P}$ and $3 \mathrm{Q}$ ) as seen in NHEM (Figure 3R). In addition, transcripts of MITF-M, which is a MITF gene-isoform known to be expressed in melanocytes and c-Kit that is a marker of melanoblasts and melanocytes were expressed in $3 \mathrm{~F}$ iPS cell derived melanocyes (Figure 3S). To compare the efficiency of melanocyte generation from $3 \mathrm{~F}-$ and $4 \mathrm{~F}-\mathrm{iPS}$ cells, the population of SILV-positive cells was compared 7 weeks after cell differentiation and the almost same differentiation efficiency was observed between $3 \mathrm{~F}$ - and 4F-iPS derived cells (Figure 3T). Finally, scatter plot analyses were performed using DNA microarray to compare the global gene expression between NHEM and melanocytes derived from 3F-iPS cells at 8 weeks 

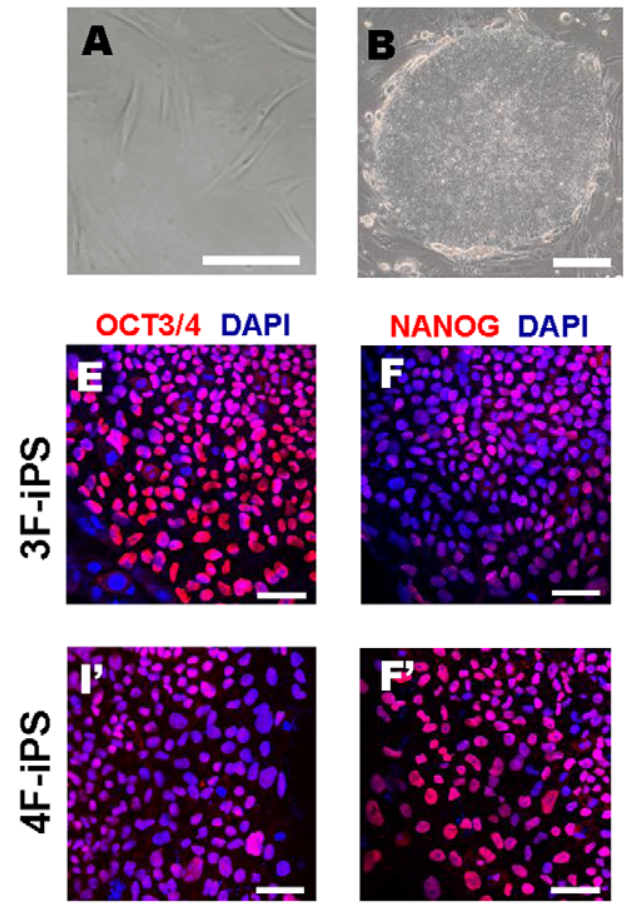

J

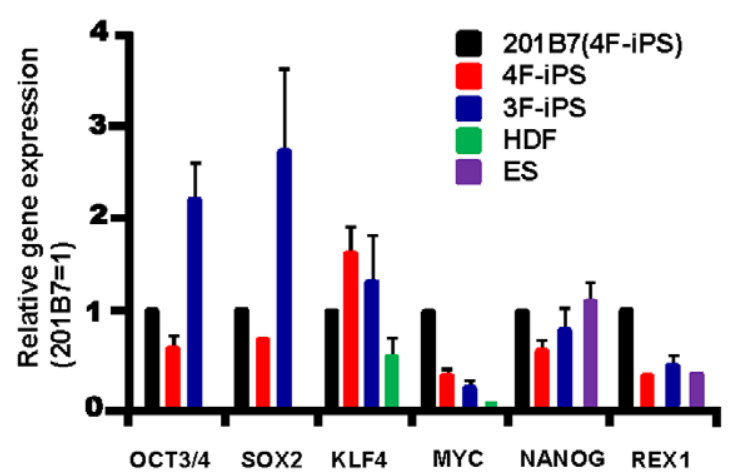

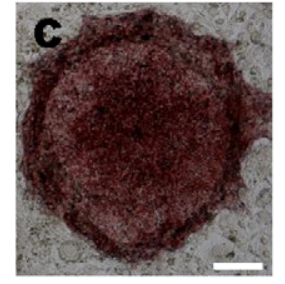
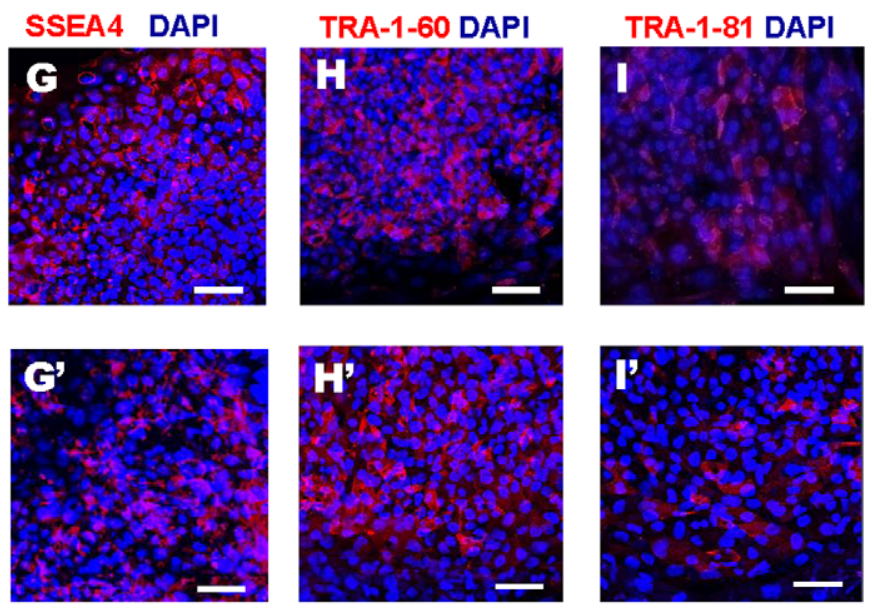

K
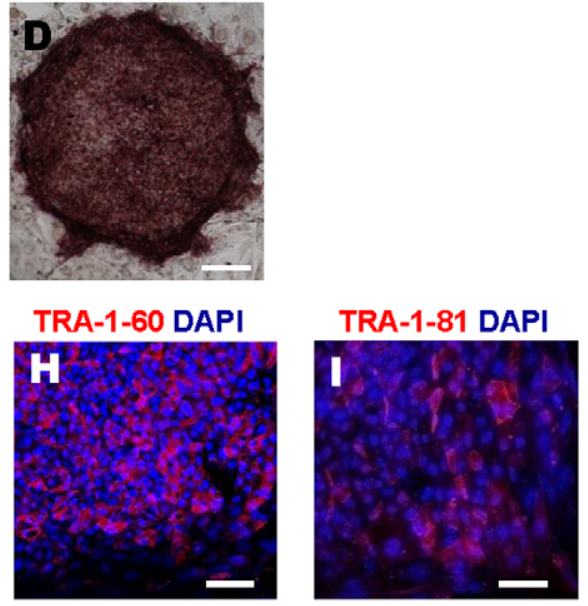
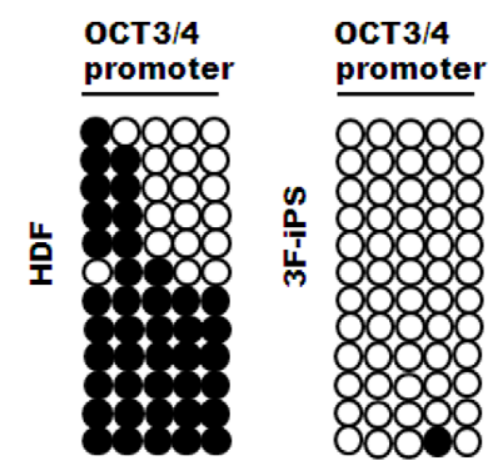

OCT3/4

promoter

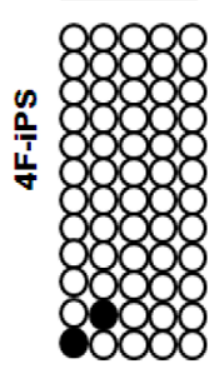

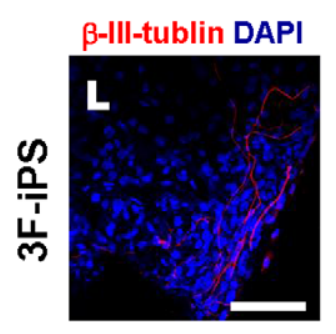
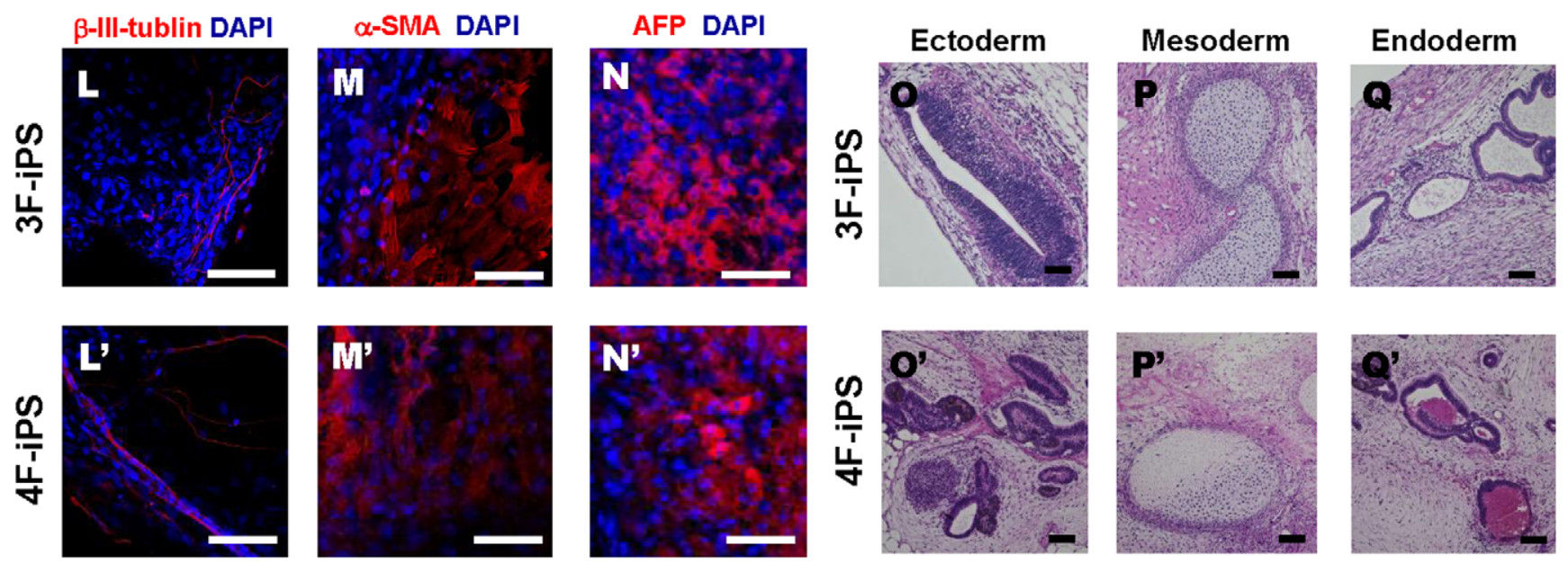
Figure 1. Induction of human 3F- and 4F-iPS cells from adult dermal fibroblasts and characterization of their pluripotency. (A) Morphology of human dermal fibroblasts. (B) Representative image of an established iPS cell colony cultured on mitomycin C-treated STO feeders. (C, D) Alkaline phosphatase (AP) staining in a 3F- (C) and 4F- (D) iPS cell colony. (E-I') Expression of pluripotency markers in established 3F-and 4F-iPS colonies. 3F-iPS (E-I) and 4F-iPS ( $\left.E^{\prime}-I^{\prime}\right)$ colonies express markers common to pluripotent cells including OCT3/4, NANOG, SSEA-4, TRA-1-60, and TRA-181. DAPI (4, 6-diamidino-2-phenylindole) staining indicates the total cell content per field. Scale bars, $20 \mu \mathrm{m}(\mathrm{A}), 100 \mu \mathrm{m}(\mathrm{B}, \mathrm{C}, \mathrm{and} \mathrm{D}), 50 \mu \mathrm{m}$ (E-I'). (J) Expression of pluripotency genes. Endogenous gene expression levels of OCT3/4, SOX2, KLF4, C-MYC, NANOG, and REX1 were determined by quantitative PCR in parental human dermal fibroblasts (HDF), 3F- and 4F-iPS cells, 201 B7 (4F-iPS cells) [20] and ES cells (KhES-1). The graphs show the average of two independent experiments. Error bar indicates mean \pm S.E.M. (K) iPS cells are demethylated at the OCT3/4 promoter relative to their parental fibroblasts. Bisulfite sequencing analysis of the OCT3/4 promoter in parental HDFs, 3F-, and 4F-iPS cells. Each horizontal row of circles represents an individual sequencing reaction for a given amplification. White circles represent unmethylated CpG dinucleotides; black circles represent methylated $\mathrm{CpG}$ dinucleotides. ( $\left(\mathrm{L}^{\prime} \mathrm{N}^{\prime}\right)$ Images of differentiated cells at day 12 . 3F- $(\mathrm{L}-\mathrm{N})$ and $4 \mathrm{~F}-\mathrm{iPS}\left(\mathrm{L}^{\prime}-\mathrm{N}^{\prime}\right)$ cells were cultured in suspension to form EBs for three weeks and then transferred to gelatin-coated plates and cultivated for another 12 days. Immunocytochemical analyses showed positive cells in spontaneously differentiated iPS cell colonies for $\beta$-III tubulin (ectoderm, $L$ and $L^{\prime}$ ), $\alpha$-smooth muscle actin ( $\alpha$-SMA, mesoderm, M and $\mathrm{M}^{\prime}$ ), and $\alpha$-fetoprotein (AFP, endoderm, $N$ and $\mathrm{N}^{\prime}$ ). Scale bar, $50 \mu \mathrm{m}$. (O-Q') Generation of teratoma-like masses in testis by xenograft of 3F- and 4FiPS. Paraffin-embedded sections were stained with hematoxylin and eosin. Resulting teratomas display features of ectoderm $\left(\mathrm{O}\right.$ and $\left.\mathrm{O}^{\prime}\right)$, mesoderm $(\mathrm{P}$ and $\left.\mathrm{P}^{\prime}\right)$ and endoderm $\left(\mathrm{Q}\right.$ and $\left.\mathrm{Q}^{\prime}\right)$. Scale bar, $100 \mu \mathrm{m}$.

doi:10.1371/journal.pone.0016182.g001

after differentiation, and a tight correlation in the gene expression profile was observed between those samples. These data strongly support that our differentiation conditions are capable of directing the generation of NHEM-like epidermal melanocytes from iPS cells (Fig. 3U).

\section{iPS cells differentiate into cells expressing neural crest} and melanocyte stem cell markers

Melanocytes are known to be derived from neural crest cells in early development and their numbers are believed to be sustained by melanocyte stem cells in dermal tissues in vivo. To identify the cell origin of iPS cell derived-melanocytes in this study, we analyzed expression of the neural crest marker Slug, and one of the putative melanocyte stem cell markers PAX3, melanoblast and melanocyte marker MITF, as well as the pluripotency marker NANOG, using quantitative RT-PCR. The expression of neural crest marker genes was observed in EB-derived cells cultured on fibronectin-coated dishes in melanocyte differentiation medium for 7 days and the gene expression of NANOG was silenced in 3F-iPS MEL (Figure 4A).
We also performed flow cytometric and immunocytochemical analyses on EB-derived cells cultured in melanocyte differentiation medium for one week. Flow cytometry showed the presence of neural crest marker p75 and HNK-1 double-positive cells, which are derived from 3F-iPS cell EBs (Figure 4B). Immune-positive cells for the neural crest markers, p75, HNK-1, and AP2 $\alpha$, were observed in migrating cells from EBs one week after differentiation (Figure 4C). The immune-positive cells for a neural crest cell marker, SOX10, [21] and a possible adult melanocyte stem cell marker, PAX3 [22,23], were also observed in the EBs-derived cells cultured in melanocyte differentiation medium for a week, indicating that the iPS-derived melanocytes may be derived through neural crest cells and melanocyte stem cells as is the case for in vivo melanocyte development (Figure 4D).

\section{Discussion}

In this study, we established an in vitro method of deriving human melanocytes from iPS cells generated using either four
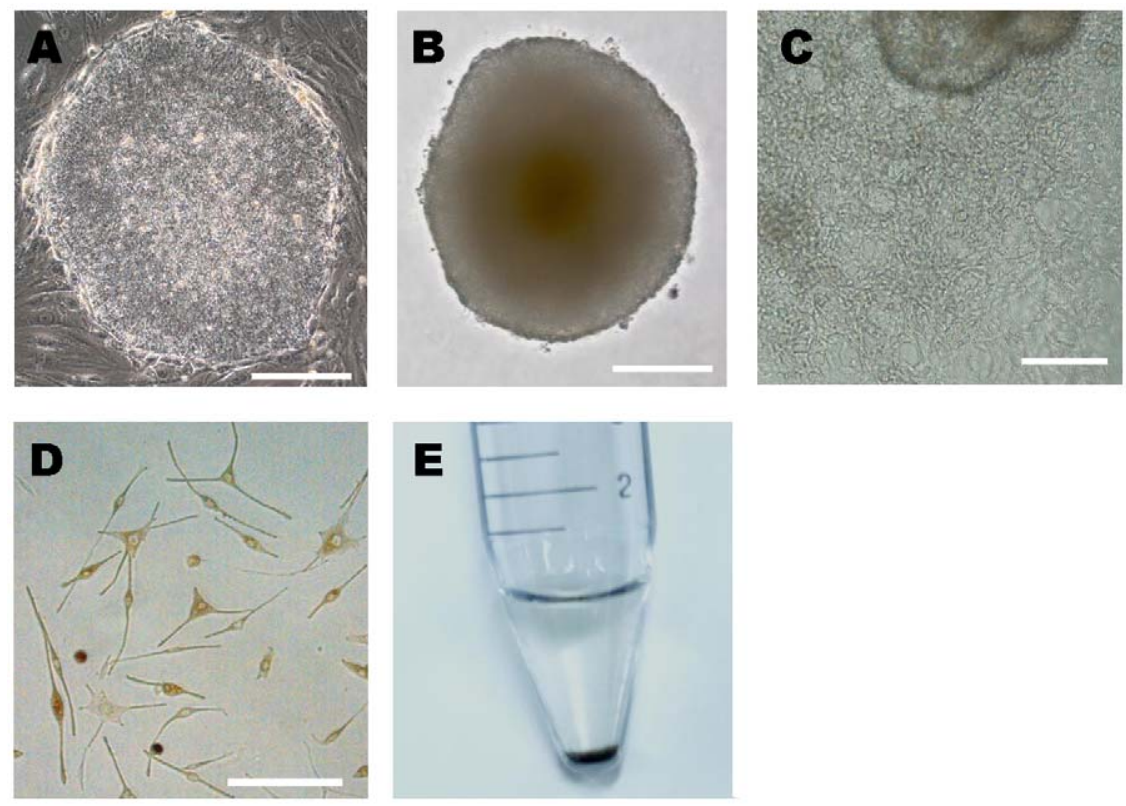

Figure 2. Generation of melanocytes from iPS cells. Efficient differentiation of human iPS cells into pigmented melanocytes in defined conditions. (A) A representative image of a 3F-iPS cell colony grown on a Mitomycin-C-treated STO feeder layer. (B) Embryoid bodies (EBs) formed in suspension under feeder-free conditions. (C) After one week in differentiation medium, cells were observed migrating out from EBs on a fibronectin-coated plate. (D) Morphology of putative melanocytes differentiated from human EBs 7 weeks after differentiation. Cells display melanocytic morphology and pigmentation. (E) Pigmented cell pellet of iPS cell-derived melanocytes 9 weeks after differentiation. Scale bar, $100 \mu \mathrm{m}(\mathrm{A}, \mathrm{B}, \mathrm{C}), 40 \mu \mathrm{m}$ (D). doi:10.1371/journal.pone.0016182.g002 

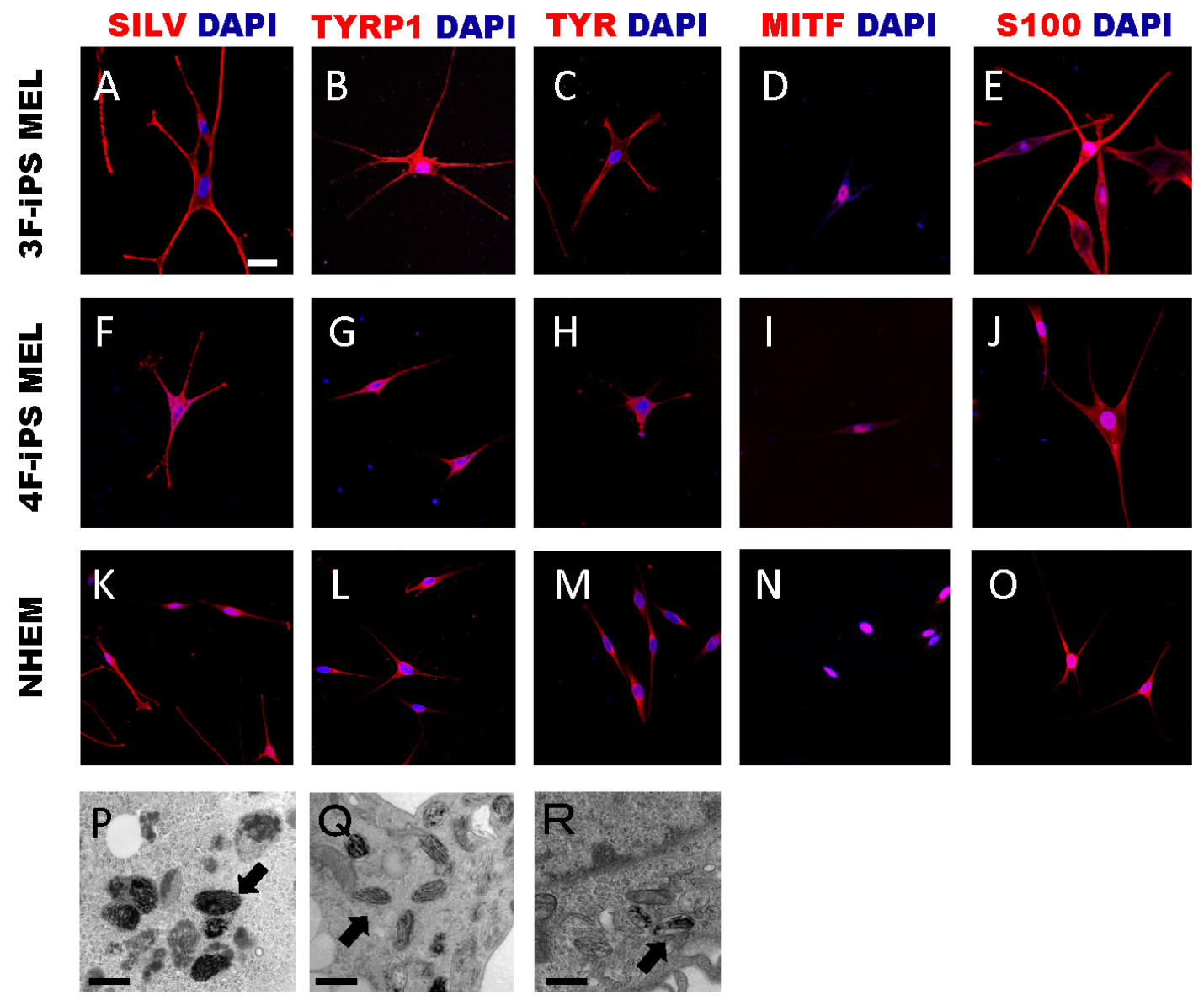

$\mathbf{S}$

$\mathbf{T}$

$\mathbf{U}$
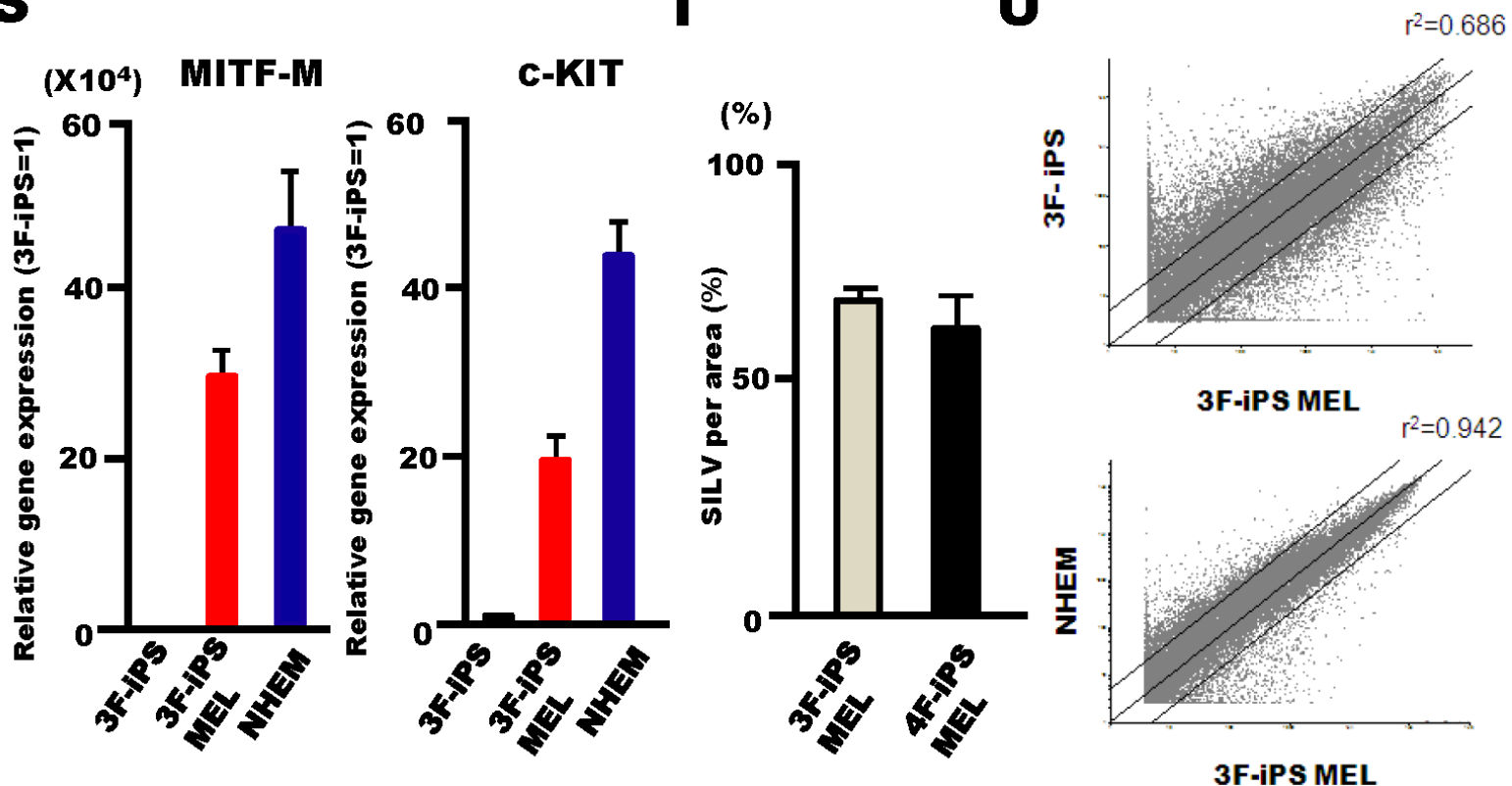

Figure 3. Marker expression characteristic of the melanocyte lineage in human iPS cell-derived melanocytes observed 8 weeks after differentiation. Either 3F- (3F-iPS MEL, A-E) or 4F- (4F-iPS MEL, F-J) iPS cells derived melanocytes were positive for an array of melanocyte markers: SILV (silver protein), TYRP1 (tyrosinase-related protein-1), TYR (tyrosinase), MITF (microphthalmia-associated transcription factor) , and S100. Normal human epidermal melanocytes (NHEM) were also stained with the same antibodies (K-O). Transmission electron microscopy images of either $3 \mathrm{~F}(\mathrm{P}), 4 \mathrm{~F}(\mathrm{Q})$-iPS cells derived melanocytes, or NHEM (R) showed that those melanocytes have many melanosomes in the cytoplasm. Scale bar, $20 \mu \mathrm{m}$ (A-O), $0.5 \mu \mathrm{m}$ (P, Q, and R). (S) Quantitative real-time PCR analysis of MITF-M and c-Kit in 3F-iPS, 3F-iPS MEL, and NHEM. The data are normalized to GAPDH and represented as fold change relative to RNA levels in 3F-iPS. The graphs show the average of two independent experiments. Error bar indicates mean \pm S.E.M. (T) Quantitation of SILV-positive cells induced from $3 \mathrm{~F}$ and $4 \mathrm{~F}$-iPS cells. Percentage of SILV positive cells upon total 
differentiation of EBs cells 7 weeks after differentiation under melanocyte differentiation medium. Error bar indicates mean \pm S.E.M ( $3 F-i P S, n=3 ; 4 F-$ iPS, $n=2)$. (U) The global gene-expression patterns were compared between human 3F-iPS cells and 3F-iPS derived-MEL (3F-iPS MEL), and between NHEM and 3F-iPS MEL. The lines indicate the linear equivalent and 5-fold differences on either side in gene expression levels between the two samples.

doi:10.1371/journal.pone.0016182.g003

(Oct3/4, SOX2, KLF4, c-MYG) or the three (without c-MYG) Yamanaka factors. Of note, deriving melanocytes from $3 \mathrm{~F}-\mathrm{iPS}$ cells should reduce the risk of tumorigenesis, based on data from a previous study [24]. Developing an in vitro human melanocyte differentiation system is important for following the reasons: 1) in addition to understanding human melanocyte biology, melanocytes generated from human iPS cells may be applied to autologous cell transplantation for patients with various pigment cell disorders such as vitiligo; 2) using patient-specific iPS cells, this in vitro system may help to reveal the mechanisms of human genetic pigment disorders or even melanoma oncogenesis, particularly through making research on MELSCs and MMSCs more accessible.

During embryonic development, the precursors of melanocytes are non-pigmented melanoblasts derived from the neural crest cells. Developmentally, embryonic melanoblasts differentiate into either mature melanocytes or MELSCs for maintaining the melanocytic system in the adult [21]. Especially in mouse, MELSCis are located in the hair follicle bulge region [7] and appear to be supported by a specialized niche [25], although MELSCis may also be located in other regions including dermis, as shown in humans. In fact, OCT4 positive-human dermal stem cells generated from foreskins lacking hair follicles have been reported to be differentiated into melanocytes [26]. Although candidate MELSC markers, DCT and PAX3, have been reported in mouse $[7,8,22]$, it is still difficult to identify MELSCs populations, especially in humans, without more specific markers. In our in vitro development system, we observed the appearance of some neural crest cell marker-positive cells one week after EBs differentiation, supporting the notion that iPS-derived melanocytes may be sequentially generated from neural crest cells, mimicking in vivo development. In addition to MELSCs [7], human skins contain various stem cell populations such as hair follicle epidermal stem cells [27], hair follicle stem cells [28], bulge neural crest-derived stem cells [29,30], and skin-derived precursors (SKPs) [31]. It may also be possible to generate such stem cell populations by modifying this culture system starting from iPS cells, eventually leading to better understanding of such human skin progenitor and/or stem cell populations.

Cancer cells including melanoma are suspected to be derived from stem/progenitor cells in which genetic alterations have accumulated [6]. Additionally, cancer stem cells, which have a high tumor initiating ability and cause relapses due to chemotherapy resistance have recently been proposed. Thus, cancer stem cells appear to be an important target for treatment [32]. It was suggested that cancer stem cells may have a similar phenotype as normal tissue stem cells in some cancers. However, the existence and phenotype of MMSCs is still controversial [33], despite various possible markers such as CD20, CD133, CD271, or ABCB5 having been previously suggested [12,34]. Identification of human MELSCs, or the generation of melanoma cells via melanoma-causing genetic alterations using this in vitro human melanocyte differentiation method from iPS, may lead to further insights into MMSC biology and subsequent development of new treatments targeting MMSCs.

It is important to establish methods to identify good quality human iPS cell clones that are free from tumor development. The safety of the melanocytes generated must still be examined by engraftment in immunodeficient mice. Additionally, based on the percentages of SILV-positive cells, our differentiated cell populations do not appear completely homogeneous. Thus, efforts to increase the purity of differentiated populations, including the removal of immature and/or undifferentiated cells that may be tumorigenic [35], are important to warrant safety prior to clinical cell therapies. In addition, to reduce the risk of tumorigenicity, use of L-MYC instead C-MYC may be an optional choice for the generation of clinical grade iPS cells [36]. In this study, we generated iPS cells using a retrovirus system, and observed imperfect transgene silencing in 3F-iPS as shown in supplemental data; thus, in the future we should develop new methods of generating clinical grade iPS cells without genomic integration of retroviral transgenes, in addition to generating homogeneous iPS clones based on the expression level of the four Yamanaka factors and NANOG.

The technique described herein is especially important for pigment cell disorders, as establishment and expansion of adult melanocytes are both challenging processes. Melanocytes from autologous iPS cells have an obvious advantage over ES cells, due to a negligible chance of immunological rejection, above and beyond surpassing ethical issues. Collectively, it may be important to find technology to select and/or increase iPS cell clones that can generate melanocytes with high yield, as each iPS cell clone may have different differentiation potency. In our culture system, the generation of neural crest cells was observed during the differentiation process. Although developmental models suggest that neural crest cells would also act as the source for melanocytes in vitro, we were unable to directly demonstrate this linearity in our differentiation system. We are now optimizing the isolation of intermediate neural crest cells using cell sorting in an attempt to increase the purity and quantity of melanocyte culture. Recently efficient methods to generate neural crest cells from human ES and iPS cells using SMAD inhibition have been reported [37]. Combining our current procedure with SMAD inhibition may ultimately increase the yield of melanocytes through the efficient induction of a transient neural crest cell population. In the future, we predict we will be able to achieve much higher efficiencies of melanocyte derivation by enrichment of either neural crest cells or MELSCs through chemical and/or physical means.

In the present study, we used Wnt3, SCF, ET3, and cAMP inducers (cholera toxin) in our melanocyte differentiation medium. MITF regulates melanocyte lineage by activating pigmentproducing genes such as DCT and tyrosinase, and contributes to cell survival by up-regulating anti-apoptotic genes $\mathrm{Bcl}-2$ and BclXL [38]. The MITF promoter is regulated by the transcription factors, PAX3, SOX10, LEF1, and CREB, all of which are downstream signaling molecules of Wnt, ET3, SCF, and cAMP [39]. Therefore, we believe our differentiation conditions bias melanocyte differentiation through a combinatorial activation of MITF. We are currently trying to improve the compositions of our differentiation medium to further increase the yield of melanocytes and reduce the number of components as more simple compositions would be desirable to generate GMP (good manufacturing practice) grade melanocytes for use in future clinical applications.

In summary, we have established in vitro differentiation system for human melanocytes from 3F- and 4F-iPS cells. Recently, many 
SLUG

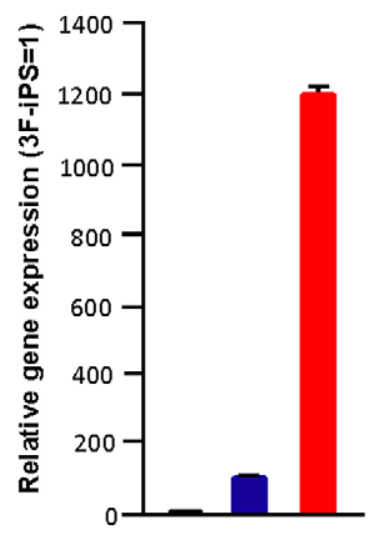

PAX3

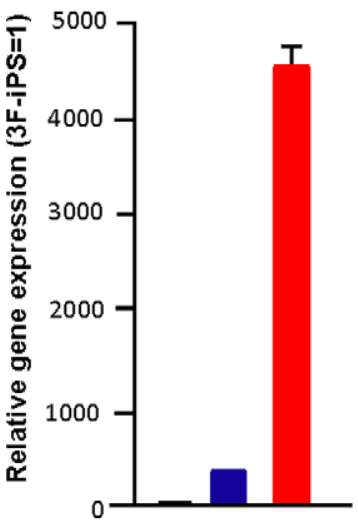

MITF

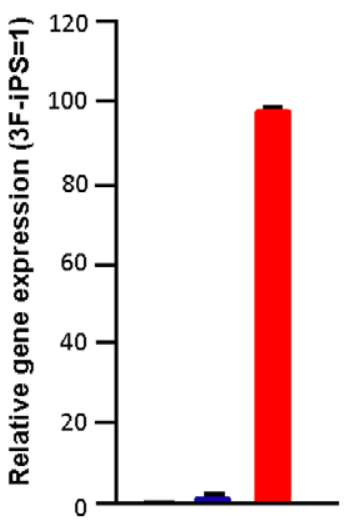

NANOG

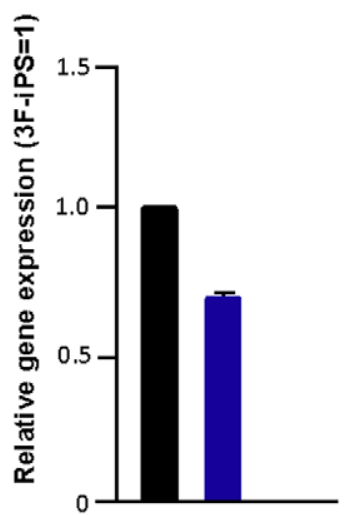

3F-iPS

EB-Diff

3F-iPS MEL
B

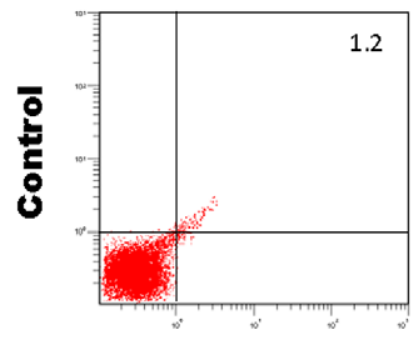

Control

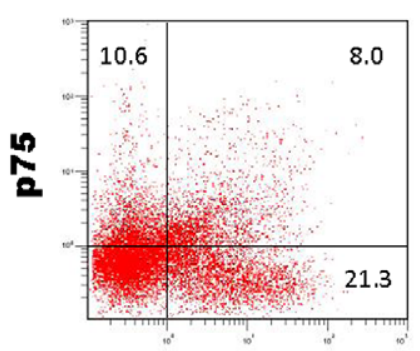

HNK-1

\section{C}
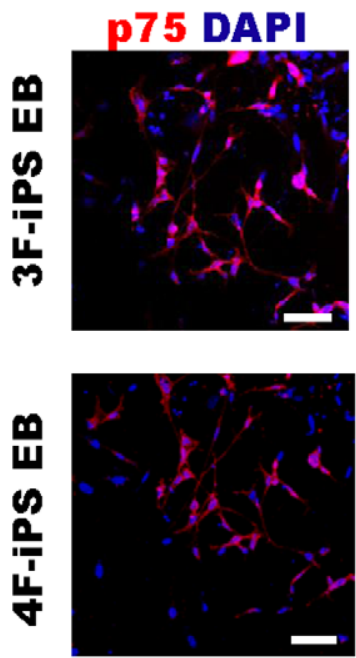
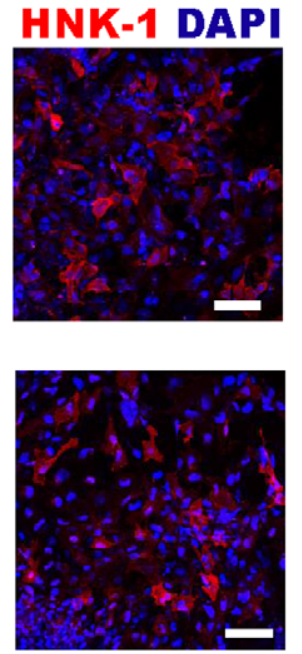
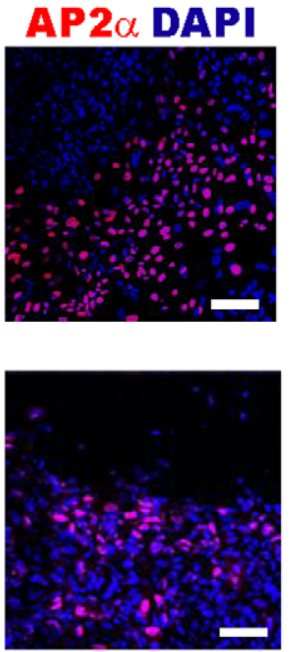

D

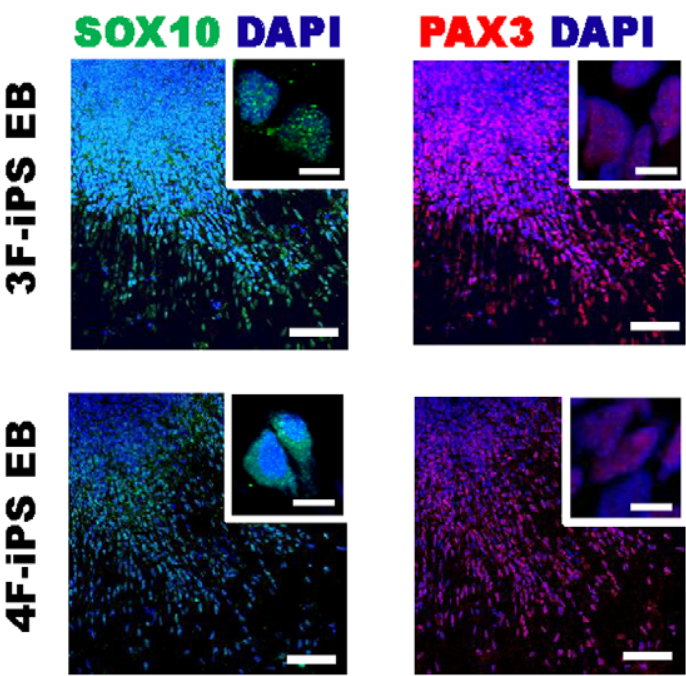

Figure 4. 3F- and 4F-iPS cells can be differentiated into NCSC and MELSCs. (A) Gene expression analysis of a neural crest stem cell marker (Slug), a melanocyte stem cell marker (PAX3), a melanocytic marker (MITF), pluripotency marker (NANOG) in 3F-iPS cells, differentiated cells derived from EBs on fibronectin-coated plates in melanocyte differentiation medium at 7 days after differentiation (EB-DIFF), and 3F-iPS derived melanocytes (3F-iPS MEL). Transcript levels were normalized to GAPDH. The graphs show the average of two independent experiments. Error bar indicates mean \pm S.E.M. (B) Representative flow cytometry results for HNK-1 and p75 staining in 3F-iPS EB-derived cells cultured in melanocyte differentiation medium for one week. Dead cells were excluded using PI staining. (C) Immunocytochemistry revealing cells positive for NCSC markers (p75, HNK-1, and AP2 $\alpha$ ) in both 3F- and 4F-iPS cells derived EB-differentiated cells 7 days after differentiation. Scale bar, 50 um. (D) Images show cells positive for neural crest cell marker SOX10, as well as possible melanocyte stem cell marker, PAX3 in both 3F- and 4F-iPS cell-derived EB-differentiated cells 7 days after differentiation. Scale bar, $50 \mu \mathrm{m}$ and $10 \mu \mathrm{m}$ (insert images). doi:10.1371/journal.pone.0016182.g004 
patient-specific human iPS cell lines have been established for diseases such as ALS, Familial dysautonomia, Parkinson's disease, and SMA [40]. If differentiated cells from patient-specific iPS cells can faithfully recapitulate the disease phenotype, iPS cell technology will contribute significantly to revealing disease mechanisms and developing drug screening systems for disease. Building on this paradigm, further analysis of the mechanism of melanocyte development using directed differentiation will contribute to not only human melanocyte biology, but also an understanding of various pigment cell disorders leading to the development of new therapeutic strategies.

\section{Materials and Methods}

\section{Generation of human induced pluripotent stem (iPS) cells and ES cell culture}

HDFs (human dermal fibroblasts) from facial dermis of a 36year-old Caucasian female (Cell Applications Inc, San Diego, CA) were used for the establishment of 4F-iPS cells (OCT3/4, SOX2, KLF4, MYG). 3F-iPS cells (OCT3/4, SOX2, KLF) were generated from primary skin fibroblasts derived from a healthy 16-year-old female volunteer by skin punch biopsy with written informed consent (Keio University School of Medicine). All fibroblasts were maintained in DMEM containing 10\% FBS. The induction of human iPS cells was performed as previously described by Takahashi et al. [20]. Briefly, fibroblasts lentivirally expressing the mouse receptor for retroviruses (Slc7al) were seeded at $3 \times 10^{5}$ cells per $60 \mathrm{~mm}$ dish, and infected the next day with retroviruses delivering all four reprogramming factors or three factors without c-MYC. Seven days after transduction, cells were harvested by trypsinization and replated at $5 \times 10^{4}-5 \times 10^{5}$ cells per $100 \mathrm{~mm}$ dish on a mitomycin C-treated STO feeder layer (DS Pharma, Osaka, Japan). The next day, the medium was replaced with human iPS medium containing FGF-2 $(4 \mathrm{ng} / \mathrm{ml}$, Wako, Osaka, Japan). The medium was changed every other day until colonies were picked. 3F- and 4F-iPS cells, and $201 \mathrm{~B} 7 \mathrm{iPS}$ cells [20] were established and characterized following methodology described in the previous study. ES cells (KhES-1) [41] were cultured on the feeder cells in iPS culture medium. Established iPS cells are available upon request to Hideyuki Okano (Keio University).

\section{Spontaneous in vitro differentiation}

Embryoid bodies (EBs) were formed from floating culture in EB medium consisting of DMEM/Ham's F12 containing 20\% Knockout Serum Replacement (Invitrogen, Carlsbad, CA), $2 \mathrm{mM}$ L-glutamine, $1 \times 10^{-4} \mathrm{M}$ nonessential amino acids, $1 \times 10^{-4} \mathrm{M} 2$-mercaptoethanol, and $0.5 \%$ penicillin and streptomycin in a bacteria dish for 3 weeks. EBs were then transferred to a gelatin-coated dish in the same iPS medium as described above.

\section{Melanocyte induction from iPS cells}

To induce melanocytic differentiation, EBs were derived as described above, however, after three weeks, EBs were plated on a fibronectin-coated dish in DMEM and MCDB 201 based differentiation medium containing $50 \mathrm{ng} / \mathrm{ml}$ Wnt3a (R\&D Systems, Minneapolis, MN), $50 \mathrm{ng} / \mathrm{ml}$ stem cell factor (SCF, R\&D Systems), $100 \mu \mathrm{M}$ Endothelin-3 (ET-3, American Peptide Company, Sunnyvale, CA), 20 pM cholera toxin (Toyobo, Osaka, Japan), 50 nM TPA (12-tetra decanoyl-phorbol 13-acetate, SigmaAldrich, St Louis, MO), 4 ng/ml FGF-2 (WAKO), $100 \mu \mathrm{M}$ Lascorbic acid (Sigma), $0.05 \mu \mathrm{M}$ dexamethasone (Sigma), $1 \mathrm{mg} / \mathrm{ml}$ linoleic acid-bovine serum albumin (Sigma), and $1 \mathrm{X}$ insulintransferrin-selenium (Sigma) following the method described by
Fang et al. [14]. Before attaining confluence, cells were dissociated using TrypLE Select (Invitrogen) and replated onto a fibronectin (BD Biosciences, San Jose, CA)-coated dish in differentiation medium until the appearance of pigmented cells and continued to be cultured for few months.

\section{Human melanocyte culture cells}

Cultured normal human foreskin-derived epidermal melanocyte (NHEM) cells were obtained from Kurabo (Osaka, Japan). Melanocytes were maintained in melanocyte complete 254 culture medium (Kurabo) supplemented with $0.5 \%$ fetal bovine serum, $5 \mu \mathrm{g} / \mathrm{ml}$ insulin, $0.5 \mu \mathrm{M}$ hydrocortisone, $5 \mu \mathrm{g} / \mathrm{ml}$ transferring, $3 \mu \mathrm{g} / \mathrm{ml}$ heparin, $3 \mathrm{ng} / \mathrm{ml}$ human FGF-2, $10 \mathrm{ng} / \mathrm{ml}$ phorbol-12myristate- 13 -acetate, and $0.2 \%$ bovine pituitary extract.

\section{Teratoma formation}

For iPS injection into the testis of 8-week-old NOD/SCID mice (OYC International, Tokyo, Japan), a $1 \mathrm{~cm}$ longitudinal incision in the abdominal wall was made, and the testis was exteriorized. One million iPS cells were inoculated into the testis parenchyma with a Hamilton pipette, and the wound was sutured. Eight weeks after injection, tumors were dissected and fixed with PBS containing $4 \%$ paraformaldehyde (PFA). Paraffin-embedded tissues were sectioned and stained with hematoxylin and eosin.

\section{RT-PCR}

Total RNA was purified with Trizol reagent (Invitrogen) and PureLink RNA Mini Kit (Invitrogen) after DNAase I treatment to remove genomic DNA. cDNA synthesis was performed using High Capacity RNA-to-cDNA Master Mix (Applied Biosystems, Forster City, CA) according to the manufacturer's instructions. The primers were designed as follows: c-Kit forward primer, 5 'GTTCTGCTCCTACTGCTTCGG-3'; reverse primer, 5' -TAACAGCGTAATCTCGTCGCG-3', MITF forward primer, 5'TGCCCAGGCATGAACACAC-3'; reverse primer, 5' ${ }^{\prime}$-TGGGAAAAATACACGCTGTGAG-3'; MITF-M forward primer, 5'-ACGTTCTCTTTGCGAGTCCATCT-3'; reverse primer, 5' GGACATGCAAGCTCAGGACT-3, PAX3 forward primer, 5' AGCGGCATCGTGAGAAGTAA-3'; reverse primer, 5'-CTTCATCTGATTGGGGTGGT-3', Slug forward primer, 5'-CATACAGCGCCATCACTGTG-3'; reverse primer, 5'-CTTGGAGGAGGTGTCAGATG-3'; GAPDH, forward primer, 5'TGAACGGGAAGCTCACTGGG-3'; reverse primer,TCGACCACGCTGTTGGTGTA-3'.

For the detection of gene expression of OCT3/4, SOC2, KLF4, c-MYG, NANOG, and REX1, primer sets published in Takahashi et al. [20] were used. The detection of transgene expression, we used sequence specific primer sets for these genes. Quantitative PCR was performed using Thunderbird syber qPCR Mix (Toyobo). Transcript levels were determined using the ABI PRISM Sequence detection System 7900HT (Applied BioSystems). Gene expression was normalized to GAPDH as an internal standard.

\section{Bisulfite Sequencing}

Genomic DNA was purified from each set of cells using a Wizard SV Genomic DNA Purification Kit (Promega, Tokyo, Japan). Bisulfite conversion was performed with $500 \mathrm{ng}$ of genomic DNA using the reagents provided in Qiagen's EpiTect Bisulfate kit (Qiagen, Tokyo, Japan). The promoter region of human OCT3/4 (-2344 to -2126$)$ was amplified using primers following the study by Freberg et al. [42]; forward primer, 5'ATTTGTTTTTTGGGTAGTTAAAGGT-3'; reverse primer, 
5'-CGAACTATGTTCATGTTAATAACATCG-3'. The PGR products were subcloned into pGEM-T Easy (Promega) and sequenced. Twelve clones of each sample were verified by sequencing with the $\mathrm{T} 7$ universal primer.

\section{DNA Microarray}

Approximately $10^{6}$ cells were used for total RNA extraction using the PureLink RNA Mini Kit (Invitrogen), according to the manufacturer's instructions. RNA quality was verified with the Bioanalyser System (Agilent Technologies, Paolo Alto, CA), using RNA Nano Chips. $1 \mu \mathrm{g}$ of RNA was processed for hybridization on the Whole Human Genome Microarray $4 \times 44 \mathrm{k}$ (Agilent). Processing was done according to the recommendations of the manufacturer. All genomic and transcriptomic analysis was carried using GeneSpring software 7.3.1. (Agilent). Signal intensities less than 0.01 were set to 0.01 , then each chip was normalized to the 50th percentile of the measurements taken from that chip.

\section{Flow cytometry}

Cells were analyzed using an EPICS XL instrument (BeckmanCoulter). Prior to flow cytometry, cells were dissociated with Accumax (Innovative Cell Technologies, San Diego, CA) for $15 \mathrm{~min}$ at room temperature and then filtrated with $40 \mu \mathrm{m}$ cell strainers. CD57 (HNK-1) FITC-conjugated antibody (BeckmanCoulter), CD271 (p75) PE- conjugated antibody (Biolegend, San Diego, CA), Mouse IgG-PE isotype antibody, and Mouse IgMFITC isotype antibody were used for immunolabeling. Propidium Iodide (PI) staining excluded dead cells.

\section{Immunocytochemical analysis and alkaline phosphatase staining}

For immunocytochemistry, cells were fixed with phosphate buffered saline (PBS) containing 4\% PFA for $20 \mathrm{~min}$ at room temperature. Then, cells were subjected to immunofluorescence staining using the following primary antibodies: $\alpha$-fetoprotein (AFP) (1:500, DAKO, Kyoto, Japan), $\alpha$-smooth muscle actin (SMA) (1:100, Sigma), AP2 $\alpha$ (1:50, Cell Signaling Technology, Beverly, MA), $\beta$-III-tubulin (1:1000, Sigma), microphthalmiaassociated transcription factor (MITF) (1:50, Thermo Scientific, Rockford, IL), HNK-1 (1:200, Millipore, Billerica, MA), NANOG (1:1000, Reprocell, Tokyo, Japan), OCT3/4 (1:200, BD Bioscience, San Diego, CA), p75 (1:100, Advanced Targeting Systems), PAX3 (1:100, R\&D Systems), S100 (1:500, DAKO), silver protein

\section{References}

1. Alkhateeb A, Fain PR, Thody A, Bennett DC, Spritz RA (2003) Epidemiology of vitiligo and associated autoimmune diseases in Caucasian probands and their families. Pigment Cell Res 16: 208-214.

2. Czajkowski R, Placek W, Drewa T, Kowaliszyn B, Sir J, et al. (2007) Autologous cultured melanocytes in vitiligo treatment. Dermatol Surg 33: 1027-1036; discussion 1035-1026.

3. van Geel N, Ongenae K, Naeyaert JM (2001) Surgical techniques for vitiligo: a review. Dermatology 202: 162-166.

4. Grichnik JM (2008) Melanoma, nevogenesis, and stem cell biology. J Invest Dermatol 128: 2365-2380.

5. Schatton T, Frank MH (2008) Cancer stem cells and human malignant melanoma. Pigment Cell Melanoma Res 21: 39-55.

6. Sabatino M, Stroncek DF, Klein H, Marincola FM, Wang E (2009) Stem cells in melanoma development. Cancer Lett 279: 119-125.

7. Nishimura EK, Granter SR, Fisher DE (2005) Mechanisms of hair graying: incomplete melanocyte stem cell maintenance in the niche. Science 307: 720-724.

8. Osawa M, Egawa G, Mak SS, Moriyama M, Freter R, et al. (2005) Molecular characterization of melanocyte stem cells in their niche. Development 132: 5589-5599.

9. Fang D, Nguyen TK, Leishear K, Finko R, Kulp AN, et al. (2005) A tumorigenic subpopulation with stem cell properties in melanomas. Cancer Res 65: 9328-9337.
(SILV) (1:50, DAKO), SOX10 (1:100, Abcam), SSEA-4 (1:200, Millipore), TRA-1-60 (1:200, Millipore), TRA-1-81 (1:200, Millipore), tyrosinase (TYR) (1:100, Santa Cruz Biothechnology, Inc., Santa Cruz, CA), tyrosinase-related protein-1 (TYRP1) (1:20, COVANCE, Princeton, NJ). After PBS washes, antibody binding was visualized using either Alexa Fluor 488 or 546-conjugated secondary antibodies (Invitrogen), and the nuclei were stained with DAPI (4',6-diamino-2-phenylindole). Images were obtained using a Zeiss LSM-510 confocal microscope (Zeiss, Tokyo, Japan). Alkaline phosphatase staining was performed using the Leukocyte Alkaline Phosphatase kit (Sigma).

\section{Electron microscopy}

Cells were fixed with $2.5 \%$ glutaraldehyde and $4 \%$ PFA in $0.1 \mathrm{M}$ cacodylate buffer ( $\mathrm{pH}$ 7.4) and post-fixed with $1 \% \mathrm{OsO}_{4}$. Images were obtained using an electron microscope (Japanese Electronic Optical Laboratories, JEOL-1230).

\section{Supporting Information}

Figure S1 Quantitative PGR for expression of retroviral transgenes in human iPS cells.

(TIF)

Figure S2 Analysis of differentiated cells in teratoma. (TIF)

\section{Acknowledgments}

The authors would like to acknowledge Dr. N. Nakatsuji (Kyoto University) for providing the KhES cells, the technical assistance of Dr. T. Nagai (Keio University) for electron microscopy studies, M. Fujiwara (Keio University) for Microarray data analysis, and Dr. Knut Woltjen (Kyoto University) for critical reading of the manuscript. We also thank the Core Instrumentation Facility, Keio University School of Medicine for technical assistance.

\section{Author Contributions}

Conceived and designed the experiments: SO HO YK. Performed the experiments: YI WA. Analyzed the data: SO YI. Contributed reagents/ materials/analysis tools: YO YM SY. Wrote the paper: SO WA HO YK. Generated and characterized the human iPS cells (WDT2 and WA29) from skin fibroblasts prepared by: MO MA. Performed the induction of human iPS cell into melanocytes and all other experiments: SO RK.
10. Monzani E, Facchetti F, Galmozzi E, Corsini E, Benetti A, et al. (2007) Melanoma contains CD133 and ABCG2 positive cells with enhanced tumourigenic potential. Eur J Cancer 43: 935-946.

11. Schatton T, Murphy GF, Frank NY, Yamaura K, Waaga-Gasser AM, et al. (2008) Identification of cells initiating human melanomas. Nature 451: 345-349.

12. Boiko AD, Razorenova OV, van de Rijn M, Swetter SM, Johnson DL, et al. Human melanoma-initiating cells express neural crest nerve growth factor receptor CD271. Nature 466: 133-137.

13. Levy C, Khaled M, Fisher DE (2006) MITF: master regulator of melanocyte development and melanoma oncogene. Trends Mol Med 12: 406-414.

14. Fang D, Leishear K, Nguyen TK, Finko R, Cai K, et al. (2006) Defining the conditions for the generation of melanocytes from human embryonic stem cells. Stem Cells 24: 1668-1677.

15. Yamane T, Hayashi S, Mizoguchi M, Yamazaki H, Kunisada T (1999) Derivation of melanocytes from embryonic stem cells in culture. Dev Dyn 216: 450-458.

16. Dimos JT, Rodolfa KT, Niakan KK, Weisenthal LM, Mitsumoto H, et al. (2008) Induced pluripotent stem cells generated from patients with ALS can be differentiated into motor neurons. Science 321: 1218-1221.

17. Ebert AD, Yu J, Rose FF, Jr., Mattis VB, Lorson CL, et al. (2009) Induced pluripotent stem cells from a spinal muscular atrophy patient. Nature 457: 277-280.

18. Lee G, Papapetrou EP, Kim H, Chambers SM, Tomishima MJ, et al. (2009) Modelling pathogenesis and treatment of familial dysautonomia using patientspecific iPSCs. Nature 461: 402-406. 
19. Soldner F, Hockemeyer D, Beard C, Gao Q Bell GW, et al. (2009) Parkinson's disease patient-derived induced pluripotent stem cells free of viral reprogramming factors. Cell 136: 964-977.

20. Takahashi K, Tanabe K, Ohnuki M, Narita M, Ichisaka T, et al. (2007) Induction of pluripotent stem cells from adult human fibroblasts by defined factors. Cell 131: 861-872.

21. White RM, Zon LI (2008) Melanocytes in development, regeneration, and cancer. Cell Stem Cell 3: 242-252.

22. Abbas O, Mahalingam M (2009) Epidermal stem cells: practical perspectives and potential uses. Br J Dermatol 161: 228-236.

23. Lang D, Lu MM, Huang L, Engleka KA, Zhang M, et al. (2005) Pax3 functions at a nodal point in melanocyte stem cell differentiation. Nature 433: 884-887.

24. Nakagawa M, Koyanagi M, Tanabe K, Takahashi K, Ichisaka T, et al. (2008) Generation of induced pluripotent stem cells without Myc from mouse and human fibroblasts. Nat Biotechnol 26: 101-106.

25. Nishimura EK, Suzuki M, Igras V, Du J, Lonning S, et al. Key roles for transforming growth factor beta in melanocyte stem cell maintenance. Cell Stem Cell 6: $130-140$

26. Li L, Fukunaga-Kalabis M, Yu H, Xu X, Kong J, et al. Human dermal stem cells differentiate into functional epidermal melanocytes. J Cell Sci 123: 853-860.

27. Jaks V, Barker N, Kasper M, van Es JH, Snippert HJ, et al. (2008) Lgr5 marks cycling, yet long-lived, hair follicle stem cells. Nat Genet 40: 1291-1299.

28. Tumbar T, Guasch G, Greco V, Blanpain C, Lowry WE, et al. (2004) Defining the epithelial stem cell niche in skin. Science 303: 359-363.

29. Amoh Y, Li L, Katsuoka K, Penman S, Hoffman RM (2005) Multipotent nestinpositive, keratin-negative hair-follicle bulge stem cells can form neurons. Proc Natl Acad Sci U S A 102: 5530-5534.

30. Yu H, Kumar SM, Kossenkov AV, Showe L Xu X Stem cells with neural crest characteristics derived from the bulge region of cultured human hairfollicles. J Invest Dermatol 130: 1227-1236.
31. Toma JG, McKenzie IA, Bagli D, Miller FD (2005) Isolation and characterization of multipotent skin-derived precursors from human skin. Stem Cells 23: 727-737.

32. Dalerba P, Cho RW, Clarke MF (2007) Cancer stem cells: models and concepts. Annu Rev Med 58: 267-284

33. Quintana E, Shackleton M, Foster HR, Fullen DR, Sabel MS, et al. Phenotypic Heterogeneity among Tumorigenic Melanoma Cells from Patients that Is Reversible and Not Hierarchically Organized. Cancer Cell 18: 510-523.

34. Zabierowski SE, Herlyn M (2008) Melanoma stem cells: the dark seed of melanoma. J Clin Oncol 26: 2890-2894.

35. Miura K, Okada Y, Aoi T, Okada A, Takahashi K, et al. (2009) Variation in the safety of induced pluripotent stem cell lines. Nat Biotechnol 27: 743-745.

36. Nakagawa M, Takizawa N, Narita M, Ichisaka T, Yamanaka S Promotion of direct reprogramming by transformation-deficient Myc. Proc Natl Acad Sci U S A 107: 14152-14157.

37. Chambers SM, Fasano CA, Papapetrou EP, Tomishima M, Sadelain M, et al. (2009) Highly efficient neural conversion of human ES and iPS cells by dual inhibition of SMAD signaling. Nat Biotechnol 27: 275-280.

38. Lin JY, Fisher DE (2007) Melanocyte biology and skin pigmentation. Nature 445: 843-850.

39. Steingrimsson E Copeland NG, Jenkins NA (2004) Melanocytes and the microphthalmia transcription factor network. Annu Rev Genet 38: 365-411.

40. Kiskinis E, Eggan K Progress toward the clinical application of patient-specific pluripotent stem cells. J Clin Invest 120: 51-59.

41. Suemori H, Yasuchika K, Hasegawa K, Fujioka T, Tsuneyoshi N, et al. (2006) Efficient establishment of human embryonic stem cell lines and long-term maintenance with stable karyotype by enzymatic bulk passage. Biochem Biophys Res Commun 345: 926-932.

42. Freberg CT, Dahl JA, Timoskainen S, Collas P (2007) Epigenetic reprogramming of OCT4 and NANOG regulatory regions by embryonal carcinoma cell extract. Mol Biol Cell 18: 1543-1553. 